International Journal of Pure and Applied Mathematics

Volume 117 No. 2 2017, 355-365

ISSN: 1311-8080 (printed version); ISSN: 1314-3395 (on-line version)

url: http://www.ijpam.eu

doi: 10.12732 /ijpam.v117i2.9

\title{
PROXIMATE AND APPROXIMATE SEQUENCES
}

\author{
Zoran Misajleski \\ Department of Mathematics \\ Faculty of Civil Engineering \\ Ss. Cyril and Methodius University \\ 9, Goce Delcev blvd., 1000 Skopje, MACEDONIA
}

\begin{abstract}
In this paper the author defines and discuses the concept of approximate sequences. First, in a separate section, he discusses proximate and approximate sequences.

After that he presents some properties of approximate sequences that are anoloqous to similar results for fundamental sequences, established in previous paper [1] titled equivalence of intrinsic shape, based on V-continuous functions and shape (N. Shekutkovski, Z. Misajleski, G. Markoski, M. Shoptrajanov, Bulletin mathematique, 2013, No. 1, 39-48). The author gives an optional definition of the function $r_{\mathcal{V}}$, with the help of intersections, which in [1] is defined using notion of depth. Also he shows that in a compact metric space there exists a cofinal sequence of finite regular coverings. In addition he shows that it is possible to choose the images of functions of approximate as subsets of the union of elements of such a sequence of coverings. Furthermore, analogue theorems of [1], which refers to approximate instead for fundamental sequences, he present and prove. Finally, the author shows that shape category constructed with the classes of approximate sequences, is equivalent with the intrinsic shape category constructed with the classes of proximate sequences.
\end{abstract}

AMS Subject Classification: $54 \mathrm{C} 56$

Key Words: shape theory, function $r \mathcal{V}$, cofinal sequence of finite coverings, proximate sequence, approximate sequence, equivalence

$\begin{array}{lrc}\text { Received: } & 2017-06-14 & \text { C } 2017 \text { Academic Publications, Ltd. } \\ \text { Revised: } & 2017-11-15 & \text { url: www.acadpubl.eu } \\ \text { Published: } & \text { December } 23,2017 & \end{array}$




\section{Introduction}

"A covering" refers to a covering consisting of open sets. Let $X$ and $Y$ be compact metric spaces. We repeat the intrinsic approaches to shape.

The following definitions and statements were given in [2]:

Definition 1. Let $\mathcal{V}$ be a finite covering of $Y$. A function $f: X \rightarrow Y$ is $\mathcal{V}$ - continuous if for every point $x \in X$, there exists a neighborhood $U_{x}$ of $x$, and $V \in \mathcal{V}$, such that $f\left(U_{x}\right) \subseteq V$.

If $f: X \rightarrow Y$ is $\mathcal{W}$-continuous, then $f: X \rightarrow Y$ is $\mathcal{V}-$ continuous if for any $\mathcal{V}$, such that $\mathcal{W} \prec \mathcal{V}$. If $\mathcal{V}$ is a finite covering of $Y$, and $V \in \mathcal{V}$, then star of $V$ is then open set $s t(\mathcal{V})=\{W \mid W \in \mathcal{V}, W \cap V \neq \emptyset\}$. We form a new covering of $s t(\mathcal{V})=\{s t(V) \mid V \in \mathcal{V}\}$.

Definition 2. The functions $f, g: X \rightarrow Y$ are $\mathcal{V}$ - homotopic, if there exists a function $F: X \times I \rightarrow Y$ such that: 1) $F$ is $s t(\mathcal{V})-$ continuous, 2) $\left.F\right|_{\partial_{I} \times X}$ is $\mathcal{V}-$ continuous and 3$) F(x, 0)=f(x)$ and $F(x, 1)=g(x)(F$ is connecting $f$ and $g$ ).

The relation of $\mathcal{V}$ - homotopy is an equivalence relation. Usually, the condition 2) of the previous condition is formulated as: $2^{\prime}$ ) there exists an neighborhood $N$ of $\partial I=\{0,1\}$ such that $\left.F\right|_{X \times N}$ is $\mathcal{V}$-continuous.

Definition 3. A cofinal sequence of finite coverings $\mathcal{V}_{1} \succ \mathcal{V}_{2} \succ \ldots \succ$ $\mathcal{V}_{n} \succ \ldots$ is a sequence of finite covering of spaces, such that for any covering $\mathcal{V}$ , there exists $n$, such that $\mathcal{V}_{n} \prec \mathcal{V}$.

In a compact metric space there exists such a sequence. This fact allows working with proximate sequences instead with proximate nets.

Definition 4. The sequence $\left(f_{n}\right)$ of functions $f_{n}: X \rightarrow Y$ is a proximate sequence from $X$ to $Y$, if there exists a cofinal sequence of finite coverings of $Y, \mathcal{V}_{1} \succ \mathcal{V}_{2} \succ \ldots \succ \mathcal{V}_{n} \succ \ldots$, and for all indexes $m \geq n, f_{n}$ and $f_{m}$ are $\mathcal{V}_{n}-$ homotopic.

In this case we say that $\left(f_{n}\right)$ is a proximate sequence over $\left(\mathcal{V}_{n}\right)$. If $\left(f_{n}\right)$ and $\left(f_{n}^{\prime}\right)$ are proximate sequences from $X$ to $Y$, than there exists a cofinal sequence of finite coverings $\mathcal{V}_{1} \succ \mathcal{V}_{2} \succ \ldots \succ \mathcal{V}_{n} \succ \ldots$ such that $\left(f_{n}\right)$ and $\left(f_{n}^{\prime}\right)$ are proximate sequences over $\left(\mathcal{V}_{n}\right)$.

Definition 5. Two proximate sequences $\left(f_{n}\right)$ and $\left(f_{n}^{\prime}\right)$ are homotopic if there exists a cofinal sequence of finite coverings $\mathcal{V}_{1} \succ \mathcal{V}_{2} \succ \ldots \succ \mathcal{V}_{n} \succ \ldots$ of $Y$, such that $\left(f_{n}\right)$ and $\left(f_{n}^{\prime}\right)$ are $\mathcal{V}_{n}-$ homotopic for all integers $n$.

We say that $\left(f_{n}\right)$ and $\left(f_{n}^{\prime}\right)$ are homotopic over $\left(\mathcal{V}_{n}\right)$. 
Let $\left(f_{n}\right): X \rightarrow Y$ be a proximate sequence over $\left(\mathcal{V}_{n}\right)$ and $\left(g_{k}\right): Y \rightarrow Z$ be a proximate sequence over $\left(\mathcal{W}_{n}\right)$. For a covering $\mathcal{W}_{k}$ of $Z$, there exists a covering $\mathcal{V}_{n_{k}}$ of $Y$ such that $g\left(\mathcal{V}_{n_{k}}\right) \prec \mathcal{W}_{k}$. Then, the composition is the proximate sequence $\left(h_{k}\right)=\left(g_{k} f_{n_{k}}\right): X \rightarrow Z$. In [2] is proven that compact metric spaces and homotopy classes of proximate sequences $\left[\left(f_{n}\right)\right]$ form the shape category i.e. isomorphic spaces in this category has the same shape.

Let $X, Y$ and $Z$, are compact metric space, that lie in the Hilbert space $Q \in A R$.

The following definitions and statements were used in [3]:

Definition 6. Approximate sequence from $X$ to $Y$ is a sequence of maps $\bar{f}_{n}: X \rightarrow Q, n \in N$, such that for every neighborhood $V$ of $Y$ in $Q$, there exist a index $n_{0}$, such that for every $n \geq n_{0}, \bar{f}_{n}$ is homotopic to $\bar{f}_{n+1}$ in $V$.

We denote approximate sequence with $\left(\bar{f}_{n}\right)_{Q}$ from $X$ to $Y$ or $\left(\bar{f}_{n}\right)$ from $X$ to $Y$. Original notion in [3] is $\left(\bar{f}_{n}: X \rightarrow Y\right)_{Q}$ or $\left(\bar{f}_{n}: X \rightarrow Y\right)$.

Definition 7. Approximate sequences $\left(\bar{f}_{n}\right)$ and $\left(\bar{f}_{n}^{\prime}\right)$ from $X$ to $Y$ are homotopic, if for every neighborhood $V$ of $Y$, there exist $n_{0} \in N$, such that $\bar{f}_{n}$ is homotopic to $\bar{f}_{n}^{\prime}$ in $V$ for all $n \geq n_{0}, n \in N$.

The relation of homotopy of approximate sequences is an equivalence relation.

Definition 8. Fundamental sequence from $X$ to $Y$ is a sequence of maps $\bar{f}_{n}: Q \rightarrow Q, n=1,2,3 \ldots$, where for every neighborhood $V$ of $Y$, there exist neighborhood $U$ of $X$ and there exist $n_{0} \in N$, such that $\left.\bar{f}_{n}\right|_{U}$ is homotopic to $\left.\bar{f}_{n+1}\right|_{U}$ in $V$ for all $n \geq n_{0}$.

Fundamental sequence is denoted with $\left(\bar{f}_{n}\right)_{Q, Q}$ from $X$ to $Y$.

Definition 9. Fundamental sequences $\left(\bar{f}_{n}\right)_{Q, Q}$ and $\left(\bar{f}_{n}^{\prime}\right)_{Q, Q}$ from $X$ to $Y$ are homotopic, if for every neighborhood $V$ of $Y$ in $Q$, there exist neighborhood $U$ of $X$ in $Q$ and $n_{0} \in N$, such that $\left.\bar{f}_{n}\right|_{U}$ is homotopic to $\left.\bar{f}_{n}^{\prime}\right|_{U}$ in $V$, for all $n \geq n_{0}, n \in N$.

The relation of homotopy of fundamental sequences is an equivalence relation.

For every approximate sequence $\left(\bar{f}_{n}\right)$ from $X$ to $Y$, because $Q$ is absolute retract, there exist fundamental sequence $\left(\hat{f}_{n}\right)_{Q, Q}$ from $X$ to $Y$, such that $\left.\hat{f}_{n}\right|_{X}=\bar{f}_{n}$. 
For every approximate sequence $\left(\bar{f}_{n}\right)$ from $X$ to $Y$ and for every fundamental sequence $\left(\overline{g_{n}}\right)_{Q, Q}$ from $Y$ to $Z$, maps $\overline{g_{n}} \overline{f_{n}}: X \rightarrow Q$, define approximate sequence $\left(\bar{g}_{n} \bar{f}_{n}\right)$ from $X$ to $Z$, which is called composition of the approximate sequences $\left(\overline{g_{n}}\right)$ and $\left(\bar{f}_{n}\right)$. If approximate sequences $\left(\bar{f}_{n}\right)$ and $\left(\bar{f}_{n}^{\prime}\right)$ from $X$ to $Y$ are homotopic, and fundamental sequences $\left(\overline{g_{n}}\right)_{Q, Q}$ and $\left(\overline{g_{n}^{\prime}}\right)_{Q, Q}$ from $Y$ to $Z$ are homotopic, then the compositions $\left(\bar{g}_{n} \bar{f}_{n}\right)$ and $\left(\bar{g}_{n}^{\prime} \bar{f}_{n}^{\prime}\right)$ from $X$ to $Z$ are homotopic. Following the last claims, it can be concluded that for every approximate sequence class $\left[\left(\bar{f}_{n}\right)\right]: X \rightarrow Y$ and every fundamental class $\left\lfloor\left(\overline{g_{n}}\right)_{Q, Q}\right\rfloor: Y \rightarrow Z$ every compositions $\left(\hat{g_{n}} \overline{f_{n}}\right)$ belong to same class, which is denoted with $\left\lfloor\left(\overline{g_{n}}\right)_{Q, Q}\right\rfloor\left[\left(\bar{f}_{n}\right)\right]$. In this way, if we take the compact metric spaces to be objects, and the classes of approximate sequences to be morphisms, then we get a new category, which is called shape $S h$.

\section{Proximate and Approximate Sequences}

All spaces in this section are compact metric spaces.

Definition 10. Covering $\mathcal{V}$ of $Y$ is regular if it satisfies the following conditions:

1) If $V \in \mathcal{V}$ then $V \cap Y \neq \emptyset$ and

2) If $U, V \in \mathcal{V}$ and $U \cap V \neq \emptyset$, then $U \cap V \in \mathcal{V}$.

Let $\mathcal{V}=\left\{V_{i} \mid i=1,2, \ldots, n\right\}$ be a regular covering of $Y$ and $\bar{f}: X \rightarrow \bigcup_{V \in \mathcal{V}} V$ is continuous function i.e. map. A function: $r_{\mathcal{V}}: \bigcup_{V \in \mathcal{V}} V \rightarrow Y$ will be defined such that the set $\bigcup_{V \in \mathcal{V}} V$ in which $\mathcal{V}$ has $n$ elements, we will divide into $2^{n}-1$ disjoint nonempty subsets the most, and in each one one element from $Y$, will be chosen. Disjoint subsets are of the type $U_{i_{1}} \cap U_{i_{2}} \cap \ldots \cap U_{i_{k}} \backslash{ }_{j_{1}, j_{2}, \ldots, j_{k+1} \in\{1,2, \ldots n\}} U_{j_{1}} \cap$ $U_{j_{2}} \cap \ldots \cap U_{j_{k}+1}$ for some combination $\left\{i_{1}, i_{2}, \ldots i_{k}\right\}$ of $n$ elements, class $k$ and all combinations $\left\{j_{1}, j_{2}, \ldots j_{k+1}\right\}$ of $n$ elements, class $k+1$. Linking will be defined inductively as follows:

n) If $y \in U_{1} \cap U_{2} \cap \ldots \cap U_{n}$ we put

$$
r_{\mathcal{V}}(y)=\left[U_{1} \cap U_{2} \cap \ldots \cap U_{n}\right]
$$

for some element $\left[U_{1} \cap U_{2} \cap \ldots \cap U_{n}\right]$ from $Y \cap U_{1} \cap U_{2} \cap \ldots \cap U_{n}$. 


$$
n-1) \text { If } y \in U_{i_{1}} \cap U_{i_{2}} \cap \ldots \cap U_{i_{n-1}} \backslash\left(U_{1} \cap U_{2} \cap \ldots \cap U_{n}\right), i_{1}, i_{2}, \ldots, i_{n-1} \in\{1,2, \ldots, n\}
$$

then we put

$$
r \mathcal{V}(y)=\left[U_{i_{1}} \cap U_{i_{2}} \cap \ldots \cap U_{i_{n-1}}\right]
$$

for some element $\left[U_{1} \cap U_{2} \cap \ldots \cap U_{n}\right]$ from $Y \cap U_{1} \cap U_{2} \cap \ldots \cap U_{n-1}$

$$
0<k<n) \text { If }
$$

$y \in U_{i_{1}} \cap U_{i_{2}} \cap \ldots \cap U_{i_{k}} \backslash \bigcup_{j_{i}, j_{2}, \ldots, j_{k+1} \in\{1,2, \ldots n\}} U_{j_{1}} \cap U_{j_{2}} \cap \ldots \cap U_{j_{k+1}}$

we put

$$
r_{\mathcal{V}}(y)=\left[U_{1} \cap U_{2} \cap \ldots \cap U_{n}\right]
$$

for some element $\left[U_{i_{1}} \cap U_{i_{2}} \cap \ldots \cap U_{i_{k}}\right]$ from $Y \cap U_{i_{1}} \cap U_{i_{2}} \cap \ldots \cap U_{i_{k}}$. Because all sets of the type

$$
U_{i_{1}} \cap U_{i_{2}} \cap \ldots \cap U_{i_{k}} \backslash \bigcup_{j_{i}, j_{2}, \ldots, j_{k+1} \in\{1,2, \ldots n\}} U_{j_{1}} \cap U_{j_{2}} \cap \ldots \cap U_{j_{k+1}},
$$

are pairwise disjoint, it follows that $r_{\mathcal{V}}$ is well defined. $r_{\mathcal{V}}$ is also $\mathcal{V}$ - continuous. We can also define $r_{\mathcal{V}}$ in the following way: $r_{\mathcal{V}}(y)=[V]$ if $V$ is the smallest set in $\mathcal{V}$ which contains $y$.

If $\mathcal{V}$ is a regular covering of $Y$ and $\bar{f}: X \rightarrow \bigcup_{V \in \mathcal{V}} V$ is a map, we can define function $f: X \rightarrow Y$ with

$$
f(x)=r_{\mathcal{V}} \bar{f}(x) \quad \text { for all } \quad x \in X .
$$

The function $f$ is well defined. Since $\bar{f}$ is continuous, the function $f$ is $\mathcal{V}$ -continuous. We will say that the function $f$ is obtained from a continuous function $\bar{f}$ and covering $\mathcal{V}$.

Example 1. Let $X$ be a compact metric space, $Y=I \times I \subseteq R^{2}, \bar{f}_{1}: X \rightarrow$ $W$ and $\bar{f}_{2}: X \rightarrow \bigcup_{i=1}^{3} V_{i}$, are maps, and $f_{1,2}^{-}: X \times I \rightarrow R^{2}$ is homotopy connecting $\bar{f}_{1}$ and $\bar{f}_{2}$, where $W=(-1,2) \times(-1,2), \mathcal{V}_{1}=\{W\}, V_{1}=\left(-\frac{1}{2}, \frac{3}{2}\right) \times\left(\frac{1}{3}, \frac{2}{3}\right)$, $V_{2}=(-1,2) \times\left(\frac{1}{3}, 2\right), V_{3}=\left(\frac{-1}{2}, \frac{3}{2}\right) \times\left(-1, \frac{2}{3}\right) V_{2}=\left\{V_{1}, V_{2}, V_{3}\right\}, 1 \equiv\left(\frac{1}{2}, \frac{1}{2}\right)$, $2 \equiv\left(\frac{3}{4}, \frac{1}{2}\right)$ and $3 \equiv\left(\frac{1}{4}, \frac{1}{2}\right)$. Then $f_{1}(x)=1$ because $\bar{f}_{1} \in W$,

$$
\begin{aligned}
& 1 \quad \bar{f}_{2}(x) \in V_{1} \\
& f_{2}(x)=\left\{\begin{array}{lll}
2 & \bar{f}_{2}(x) \in V_{2} \backslash V_{1} & \text { for } x \in X \text { and }
\end{array}\right. \\
& 3 \quad \bar{f}_{2}(x) \in V_{3} \backslash V_{1} \\
& 1 \bar{f}_{1,2}(x, t) \in V_{1}, t \in[0,1) \quad \text { and } \quad \bar{f}_{1,2}(x, 1) \in V_{1} \\
& f_{1,2}(x, t)= \begin{cases}2 & \bar{f}_{1,2}(x, t) \in V_{2} \backslash V_{1} \\
3 & \bar{f}_{1,2}(x, t) \in V_{3} \backslash V_{1}\end{cases}
\end{aligned}
$$


So, $f_{1,2}(x, 0)=f_{1}(x)$ and $f_{1,2}(x, 1)=f_{2}(x)$ i.e. $f_{1,2}$ is connecting $f_{1}$ and $f_{2}$. Function $f_{2}$ is $\mathcal{V}_{2}$-continuous. Namely, if $\bar{f}_{2}(x) \in V_{1},\left(\bar{f}_{2}\right.$ is a map), there exists a neighborhood $U_{1}$ in $X$, such that $x \in U_{1}$ and $\bar{f}_{2}\left(U_{1}\right) \subseteq V_{1}$. It follows $f_{2}\left(U_{1}\right)=\{1\} \subseteq V_{1}$. If $\bar{f}_{2}(x) \in V_{2} \backslash V_{1}$, there exists a neighborhood $U_{2}$ in $X$, such that $x \in U_{2}$ and $\bar{f}_{2}\left(U_{2}\right) \subseteq V_{2}$. It follows $f_{2}\left(U_{2}\right)=\{1,2\} \subseteq V_{2}$. If $\bar{f}_{2}(x) \in V_{3} \backslash V_{1}$, there exists $U_{3}$ in $X$, such that $x \in U_{3}$ and $\bar{f}_{2}\left(U_{3}\right) \subseteq V_{3}$. It follows $f_{2}\left(U_{3}\right)=\{1,3\} \subseteq V_{3}$. Clearly the functions $f_{1}$ and $f_{1,2}$ are $\mathcal{V}_{1}$ continuous. Function $f_{1,2}$ will be homotopy as well as when $f_{1,2}(x, t)=1$, for every $(x, t) \in X \times I$.

Definition 11. Cofinal sequence of finite regular coverings is cofinal sequence of finite coverings whose coverings are regular.

Theorem 12. In a compact metric space, there exists a cofinal sequence of finite regular coverings of the space.

Proof. In a compact metric space $Y$ embedded in a Hilbert cube, there exists a cofinal sequence of finite coverings $\mathcal{V}_{1} \succ \mathcal{V}_{2} \succ \ldots \succ \mathcal{V}_{n} \succ \ldots$. On each of the coverings $\mathcal{V}_{n}, n \in N$ we add intersections of its elements. Then from every covering $\mathcal{V}_{n}, n \in N$, we reject the elements whose intersection with $Y$ is an empty set. The cofinality is not violated because if one element has empty intersection with $Y$, then all sets $V_{m}$ from $\mathcal{V}_{m}, m>n$ which are contained in some sets in $\mathcal{V}_{n}$, also have an empty intersection with $Y$. In that way we get a cofinal sequence of finite regular coverings of $Y$.

Theorem 13. For the approximate sequence $\left(\bar{f}_{n}\right)$ from $X$ to $Y$, there exists a cofinal sequence of finite regular coverings $\mathcal{V}_{1} \succ \mathcal{V}_{2} \succ \ldots \succ \mathcal{V}_{n} \succ \ldots$ of $Y$ in $Q$ such that $\operatorname{Im} \bar{f}_{n}, \operatorname{Im} \bar{f}_{n+1} \subseteq \bigcup_{V \in \mathcal{V}_{n}} V$.

Proof. From Theorem 12 it follows that there exists a cofinal sequence of finite regular coverings of $\mathcal{V}_{1}^{\prime} \succ \mathcal{V}_{2}^{\prime} \succ \ldots \succ \mathcal{V}_{n}^{\prime} \succ \ldots$ of $Y$ in $Q$. Because $Y$ is metric space, we can choose a sequence of neighborhoods $V_{n}$ of $Y$ in $Q$ such that $d\left(Y, Q \backslash V_{n}\right)<\frac{1}{n}$.

For neighborhood $V_{1}$ of $Y$ there exists number $n_{1} \in N$ such that for all $n \geq n_{1}$ there exists a homotopy $\bar{f}_{n, n+1}: X \times I \rightarrow V_{1}$ which is connecting $\bar{f}_{n}$ and $\bar{f}_{n+1}$. We are choosing $\mathcal{V}_{1}=\mathcal{V}_{2}=\ldots=\mathcal{V}_{n_{1}-1}=\{Q\}$.

For neighborhood $V_{2}$ of $Y$ there exists a number $n_{2}>n_{1}, n_{2} \in N$ such that for all $n \geq n_{2}$ there exists a homotopy $\bar{f}_{n, n+1}: X \times I \rightarrow V_{2}$ which is connecting 
$\bar{f}_{n}$ and $\bar{f}_{n+1}$. We are choosing $\mathcal{V}_{n_{1}}=\mathcal{V}_{n_{1}+1}=\ldots=\mathcal{V}_{n_{2}-1}=\left\{V_{1} \cap \mathcal{V}_{n_{1}}^{\prime}\right\}$, where $V_{1} \cap \mathcal{V}_{n_{1}}^{\prime}=\left\{V_{1} \cap V_{n_{1}}^{\prime} \mid V_{n_{1}}^{\prime} \in \mathcal{V}_{n_{1}}^{\prime}\right\}$

Continuing inductively we get that for neighborhood $V_{k}$ of $Y$ there exists a number $n_{k}>n_{k-1}, n_{k} \in N$ such that for all $n \geq n_{k}$ there exists a homotopy $\bar{f}_{n, n+1}: X \times I \rightarrow V_{k}$ which is connecting $\bar{f}_{n}$ and $\bar{f}_{n+1}$. We are choosing $\mathcal{V}_{n_{k-1}}=$ $\mathcal{V}_{n_{k-1}+1}=\ldots=\mathcal{V}_{n_{k}-1}=\left\{V_{k-1} \cap \mathcal{V}_{n_{k-1}}^{\prime}\right.$. In that way we get a cofinal sequence of finite regular coverings $\mathcal{V}_{1} \succ \mathcal{V}_{2} \succ \ldots \succ_{n} \succ \ldots$ of $Y$ with property images of maps $\bar{f}_{n, n+1}: X \rightarrow Q$ from approximate sequence $\left(\bar{f}_{n}\right)$ to be in $\bigcup_{V \in \mathcal{V}_{n}} V$ i.e. $\operatorname{Im} \bar{f}_{n, n+1} \subseteq \bigcup_{V \in \mathcal{V}_{n}} V$ for every $n \in N$. Specially $\operatorname{Im} \bar{f}_{n} \subseteq \bigcup_{V \in \mathcal{V}_{n}} V$, for every $n \in N$.

Similarly, for two approximate sequences $\left(\bar{f}_{n}\right)$ and $\left(\bar{f}_{n}^{\prime}\right)$ from $X$ to $Y$, there exists a cofinal equence of finite regular coverings $\mathcal{V}_{1} \succ \mathcal{V}_{2} \succ \ldots \succ \mathcal{V}_{n} \succ \ldots$ of $Y$ in $Q$ such that images of functions $\bar{f}_{n}$ and $\bar{f}_{n}^{\prime}$ are subsets of $\bigcup_{V \in \mathcal{V}_{n}} V$. Next theorem is proven in [1].

Theorem 14. If $Y$ is compact metric space embedded in Hilbert cube $Q$, $\mathcal{V}$ and $\mathcal{W}$ are regular coverings of $Y$ in $Q$ such that $\mathcal{W} \prec \mathcal{V}$ and $Z=\bigcup_{W \in \mathcal{W}} W$. Then $\left.r_{\mathcal{V}}\right|_{Z}$ (the restriction of $r_{\mathcal{V}}$ to $Z$ ) and $r_{\mathcal{W}}$ are $\mathcal{V}$-homotopic.

Proof. Let us consider the function $R: Z \times I \rightarrow Y$ defined by

$$
R(x, t)= \begin{cases}r_{\mathcal{V}}(x) & (x, t) \in Z \times[0,1) \\ r_{\mathcal{W}}(x) & (x, 1) \in Z \times\{1\} .\end{cases}
$$

If $(x, t) \in Z \times[0,1)$ then $R(x, t)=r_{\mathcal{V}}(x)$ and $R$ is $\mathcal{V}$-continuous in $(x, t)$.

If $(x, 1) \in Z \times\{1\}$, then $R(x, 1)=r_{\mathcal{W}}(x)=[W]$, where $W$ is the smallest set in $\mathcal{W}$ that contains $x$. From $\mathcal{W} \prec \mathcal{V}$, it follows that $W \subseteq V \in \mathcal{V}$ and we can choose $V$ to be the smallest set in $\mathcal{V}$, with the property $W \subseteq V$. Then $r_{\mathcal{V}}(V) \in V \cap Y$ and

$$
R(W \times 1)=r_{\mathcal{W}}(W) \in W \cap Y \subset V \cap Y .
$$

We consider the neighborhood $W \times[0,1]$ of $(x, 1)$ and $(w, t) \in W \times[0,1)$. There is a smallest set $V_{\mathcal{W}}$ in $\mathcal{V}$ such that $w \in V_{\mathcal{W}}$. So it follows that: $R(w, t)=$ $r_{\mathcal{V}}(w)=\left[V_{\mathcal{W}}\right] \in V_{\mathcal{W}} \cap Y$, for all $t \in[0,1)$. Also, $V_{\mathcal{W}} \subseteq V$ for all $x \in W$. Then, it follows

$$
R(W \times[0,1)) \subseteq V \cap Y .
$$


Finally from (1) and (2),

$$
R(W \times[0,1])=R(W \times[0,1)) \cup R(W \times 1) \subseteq V \cap Y
$$

it follows that $R$ is $\mathcal{V}$-continuous at $(x, 1) \in Z \times\{1\}$ and $R(x, 0)=r_{\mathcal{V}}(x)$, $R(x, 1)=r_{\mathcal{W}}(x)$.

Theorem 15. Let $\left(\bar{f}_{n}\right)$ from $X$ to $Y$ be an approximate sequence over cofinal sequence of finite regular covering $\mathcal{V}_{1} \succ \mathcal{V}_{2} \succ \ldots \succ \mathcal{V}_{n} \succ \ldots$, let $f_{n}: X \rightarrow$ $\bigcup_{V \in \mathcal{V}_{n}} V, n \in N$ be functions defined by $f_{n}=r_{\mathcal{V}_{n}} \bar{f}_{n}$ and let $f_{n, n+1}: X \times I \rightarrow$ $\bigcup_{V \in \mathcal{V}_{n}} V, n \in N$ be $f_{n, n+1}=r_{\mathcal{V}_{n}} \bar{f}_{n, n+1}$. Then the sequence $\left(f_{n}\right)$ is a proximate sequence from $X$ to $Y$.

Proof. The existence of cofinal sequence of finite regular covering $\mathcal{V}_{1} \succ$ $\mathcal{V}_{2} \succ \ldots \succ \mathcal{V}_{n} \succ \ldots$, such that $\operatorname{Im} \bar{f}_{n}, \operatorname{Im} \bar{f}_{n, n+1} \subseteq \bigcup_{V \in \mathcal{V}_{n}} V$ is provided by Theorem 12 and Theorem 13. The functions $f_{n}$ and $f_{n, n+1}$ are $\mathcal{V}_{n}$-continuous by construction and

$$
\begin{gathered}
f_{n, n+1}(x, 0)=r_{\mathcal{V}_{n}} \bar{f}_{n, n+1}(x, 0)=r_{\mathcal{V}_{n}} \bar{f}_{n}(x)=f_{n}(x), \\
f_{n, n+1}(x, 1)=r_{\mathcal{V}_{n}} \bar{f}_{n, n+1}(x, 1)=r_{\mathcal{V}_{n}} \bar{f}_{n+1}(x) .
\end{gathered}
$$

Taking into consideretion the fact that $\mathcal{V}_{n+1} \prec \mathcal{V}_{n}$ from the theorem 14 it follows that $r_{\mathcal{V}_{n}} \mid \bigcup_{V \in \mathcal{V}_{n+1}} V$ (the restriction of $r_{\mathcal{V}_{n}}$ to $\bigcup_{V \in \mathcal{V}_{n}}$ ) and $r_{\mathcal{V}_{n+1}}$ are $\mathcal{V}_{n}$-homotopic, by a homotopy $R: \bigcup_{V \in \mathcal{V}_{n+1}} V \times I \rightarrow Y$ i.e.

$$
R(x, 0)=r_{\mathcal{V}_{n}}(x), \quad R(x, 1)=r_{\mathcal{V}_{n+1}}(x), \quad \text { for all } \quad x \in \bigcup_{V \in \mathcal{V}_{n+1}} V
$$

So, the $\mathcal{V}_{n}$-homotopy $R \bar{f}_{n+1}: X \times I \rightarrow Y$ satisfies

$$
\begin{gathered}
R \bar{f}_{n+1}(x, 0)=r_{\mathcal{V}_{n}} \bar{f}_{n+1}(x) \\
R \bar{f}_{n+1}(x, 1)=r_{\mathcal{V}_{n+1}} \bar{f}_{n+1}(x)=f_{n+1}(x) .
\end{gathered}
$$

Since $\mathcal{V}_{n}$-homotopy is an equivalence relation by (3) and (4) it follows that $f_{n}(x)$ and $f_{n+1}(x)$ are $\mathcal{V}_{n}$-homotopic.

Therefore $\left(f_{n}\right)$ is a proximate sequence over $\mathcal{V}_{1} \succ \mathcal{V}_{2} \succ \ldots \succ \mathcal{V}_{n} \succ \ldots$ 
Theorem 16. If two approximate sequences $\left(\bar{f}_{n}\right)$ and $\left(\bar{f}_{n}^{\prime}\right)$ from $X$ to $Y$ are homotopic, then the obtained from the proximate sequences $\left(f_{n}\right)$ and $\left(f_{n}^{\prime}\right)$ from $X$ to $Y$ are also homotopic.

Proof. Let the approximate sequences $\left(\bar{f}_{n}\right)$ and $\left(\bar{f}_{n}^{\prime}\right)$ be homotopic with homotopy $\bar{F}_{n}: X \times I \rightarrow \bigcup_{V \in \mathcal{V}_{n}} V$ i.e. $\bar{F}_{n}(x, 0)=\bar{f}_{n}(x)$ and $\bar{F}_{n}(x, 1)=\bar{f}^{\prime}{ }_{n}(x)$. We define $F_{n}: X \times I \rightarrow Y$ by

$$
F_{n}(x, t)=r_{\mathcal{V}_{n}} \bar{F}_{n}(x, t) .
$$

Then $F-n$ is $\mathcal{V}_{n}$-continuous and $s t\left(\mathcal{V}_{n}\right)$-continuous for all points of $X \times \partial I$ and

$$
F_{n}(x, 0)=r_{\mathcal{V}_{n}} \bar{f}_{n}(x)=f_{n}(x), \quad F_{n}(x, 1)=r_{\mathcal{V}_{n}} \bar{f}_{n}^{\prime}(x)=f_{n}^{\prime}(x) .
$$

Following the previous theorem we can define the function $\Phi_{X, Y}$ from the classes of continuous functions from $X$ to $Y$ to the classes of $\mathcal{V}$-continuous functions from $X$ to $Y$, i.e $\Phi_{X, Y}\left[\left(\bar{f}_{n}\right)\right]=\left[\left(f_{n}\right)\right]$ for every class of approximate sequences $[(\bar{f})]$ from $X$ to $Y$.

We will describe a functor $\Phi: S H \rightarrow I n S h$. The functor is consists of:

1) Function $\Phi: C P t(Q) \rightarrow C p t(Q)$ defined with $\Phi(X)=X$, for every compact metric space $X$.

2) Functions $\Phi: M_{\operatorname{sor}_{S h}}(X, Y) \rightarrow \operatorname{Mor}_{I n S h}(X, Y)$ for every compact metric spaces $X$ and $Y$ defined with $\Phi\left(\left[\left(\bar{f}_{n}\right)\right]\right)=\Phi_{X Y}\left[\left(\bar{f}_{n}\right)\right]$ for every class of approximate sequences $\left[\left(\bar{f}_{n}\right)\right]$ from $X$ to $Y$.

In order to prove that $\Phi$ is a functor, we have to prove the following theorem.

Theorem 17. If $\left(\bar{f}_{n}\right)$ from $X$ to $Y$ and $\left(\bar{g}_{n}\right)$ from $Y$ to $Z$ are two approximate sequences, then

1) $\Phi\left(\left[\left(\bar{g}_{n}\right)(\bar{f})\right]\right)=\Phi\left(\left[\left(\bar{g}_{n}\right)\right]\right) \Phi\left(\left[\left(\bar{f}_{n}\right)\right]\right)$;

2) $\Phi\left(\left[\left(\overline{1}_{X}\right)\right]\right)=1_{\Phi(X)}$.

Proof. 1) Let a proximate sequence $\left(g_{n}\right)$ from $Y$ to $Z$ be obtained from approximate sequence $\left(\bar{g}_{n}\right)$, with the cofinal sequence of finite regular coverings $\left(\mathcal{W}_{k}\right)$ of $Z$ in $Q$.

Suppose $\left(f_{n_{k}}\right)$ from $X$ to $Y$ is a proximate subsequence of the proximate sequence $\left(f_{n}\right)$ obtained from approximate sequence $\left(\bar{f}_{n}\right)$. The subsequence is 
chosen such that $f_{n_{k}}\left(\mathcal{V}_{n_{k}}\right) \prec \mathcal{W}_{k}$.

The approximate sequences $\left(\bar{f}_{n}\right)$ and $\left(\bar{f}_{n_{k}}\right)$ belong to the same class. If we take into consideration a theorem from [2], then $\left(f_{n_{k}}\right)$ and $\left(f_{n}\right)$ belong to the same class and if $\bar{g}_{k} \bar{f}_{n_{k}}=\bar{h}_{k}$ we have to prove

$$
\left\lfloor r_{\mathcal{W}_{k}} \bar{h}_{k}\right\rfloor=\left\lfloor\left(r_{\mathcal{W}_{k}} \bar{g}_{k}\right)\left(r_{\mathcal{V}_{n_{k}}} \bar{f}_{n_{k}}\right\rfloor\right.
$$

i.e. $r_{\mathcal{W}_{k}} \bar{h}_{k}$ and $\left(r_{\mathcal{W}_{k}} \bar{g}_{k}\right)\left(r_{\mathcal{V}_{n_{k}}} \bar{f}_{n_{k}}\right)$ are homotopic.

Let $x \in X$. By definition

$$
r_{\mathcal{W}_{k}} \bar{h}_{k}(x)=[W]
$$

where $W$ is the smallest set $W$ in $\mathcal{W}$ such that $\bar{g}_{k} \bar{f}_{n_{k}}(x)=\bar{h}_{k}(x) \in W$. Since $\left(\bar{g}_{k} \mathcal{V}_{n_{k}}\right) \prec \mathcal{W}_{k}$ there exists $V^{\prime}$ in $\mathcal{V}$ such that $\bar{g}_{k}\left(V^{\prime}\right) \subseteq W$. On the other hand, by definition

$$
r_{\mathcal{V}_{n_{k}}} \bar{f}_{n_{k}}(x)=[V]
$$

where $V$ is the smallest set containing $x$, then $V \subseteq V^{\prime}$ and it follows $\bar{g}_{k}(V) \subseteq W$. Then

$$
\left(r_{\mathcal{W}_{k}} \bar{g}_{k}\right)\left(r_{\mathcal{V}_{n_{k}}} \bar{f}_{n_{k}}\right)(x)=r_{\mathcal{W}_{k}}\left(\bar{g}_{k}[V]\right) \in W .
$$

Then, from (5) and (6) $r_{\mathcal{W}_{k}} \bar{h}_{k}=h_{k}$ and $\left(r_{\mathcal{W}_{k}} \bar{g}_{k}\right)\left(r_{\mathcal{V}_{n_{k}}} \bar{f}_{n_{k}}\right)=g_{k} f_{n_{k}}$ are $\mathcal{W}_{k}$-near and since $h_{k}$ is $\mathcal{W}_{k}$-continuous, by Lemma 1.1 from [4], we get that $h_{k}$ and $g_{k} f_{n_{k}}$ are $\mathcal{W}_{k}$-homotopic.

2)One representive of the identical morphism in $S h$ is the class of approximate sequences, $\left(\overline{1}_{n}\right)$ from $X$ to $X$, where $\overline{1}_{n}: X \rightarrow \bigcup_{V \in \mathcal{V}_{n}} V, n \in N$ defined with $\overline{1}_{n}(x)=x, x \in X$ are identical maps in $X$. Then $\Phi\left(\left[\left[\overline{1}_{n}\right)\right]\right)=\left[\left(1_{n}\right)\right]$, where $1_{n}: X \rightarrow X, n \in N$ are copies of the identical map. $\left[\left(1_{n}\right)\right]$ is also the identical morphism in $I n S h$, since for proximate sequences $\left(f_{n}\right)$ from $X$ to $Y$ and $\left(g_{n}\right)$ from $Y$ to $X$ it is true that $\left(f_{n}\right)\left(1_{n}\right)=\left(f_{n}\right)$ and $\left(1_{n}\right)\left(g_{n}\right)=\left(g_{n}\right)$. It follows $\Phi\left(\left[\left(1_{X}\right)\right]\right)=1_{\Phi(X)}$.

The next theorem is also valid.

Theorem 18. The functor $\Phi$ is a bijection.

Proof. 1) Suppose $\left(g_{n}\right)$ is a proximate sequence. We will reformulate of theorem 1 in [7]: For every proximate sequence $\left(g_{n}\right)$ from $X$ to $Y$ there exist an approximate sequence $\left(\bar{f}_{n}\right)$ from $X$ to $Y$ and a cofinal sequence of coverings $\mathcal{V}_{1} \succ \mathcal{V}_{2} \succ \ldots \succ \mathcal{V}_{n} \succ \ldots$ such that $\bar{f}_{n}$ and $g_{n}$ are $\mathcal{V}_{n}$-close for all integers. All 
approximate sequences obtained from $\left(f_{n}\right)$ are also homotopic.

One proximate sequence $\left(f_{n}\right)$ obtained from approximate sequence $\left(\bar{f}_{n}\right)$ consists of $\mathcal{V}_{n}$-close functions $f_{n}$ and $\bar{f}_{n}$. Therefore $\left(f_{n}\right)$ and $\left(g_{n}\right)$ are homotopic and it follows that $\Phi\left[\left(\bar{f}_{n}\right)\right]=\left[\left(g_{n}\right)\right]$, i.e. the functor is surjective.

2) Suppose the proximate sequences $\left(f_{n}\right)$ and $\left(f_{n}^{\prime}\right)$ from $X$ to $Y$ are obtained from approximate sequences $\left(\bar{f}_{n}\right)$ and $\left(\bar{f}_{n}^{\prime}\right)$ from $X$ to $Y$, respectively. Suppose $\left(f_{n}\right)$ and $\left(f_{n}^{\prime}\right)$ are homotopic, i.e. $f_{n}$ and $f_{n}^{\prime}$ are connected by homotopy $F_{n}$ connecting $\bar{f}_{n}$ and $\bar{f}_{n}^{\prime}$ for all natural numbers $n$. If we take into consideration a theorem 1 from [4], it follows that there exists a continuous homotopy $\left(\bar{F}_{n}\right)$ connecting $\left(\bar{f}_{n}\right)$ and $\left(\bar{f}_{n}^{\prime}\right)$ for all natural numbers $n$, i.e. the functor is injective.

According to the previous theorems it follows that shape categories constructed with the classes of approximate and proximate sequences are equivalent.

\section{References}

[1] N. Shekutkovski, Z. Misajleski, G. Markoski, M. Shoptrajanov, Equvalence of intristic shape, based on $V$-continuous functions and shape, Bulletin mathematique, 1, (2013), $39-48$.

[2] N. Shekutkovski, Intristic definition of strong shape for compact metric spaces, Topology Proceedings, 39, (2012), 27-39.

[3] K. Borsuk, Teorija shejpov, Izdateljstvo Mir, Moskva, (1976).

[4] J. M. R. Sanjurjo, A non continuous description of the shape, Quart. J. Math. Oxford Ser., 40, (1989), 351-359.

[5] N. Shekutkovski, Z. Misajleski, Intristic shape based on $\varepsilon$-continuity and on continuiuty up to a covering are equivalent, Proceedings of FMNS 2011, 1, (2011), 77-82.

[6] N. Shekutkovski, Z. Misajleski, Intristic shape based on $\varepsilon$-continuity and on contiuity up to a covering are equivalent (II), Proceedings of FMNS 2013, 1, (2013), 87-94.

[7] N. Shekutkovski, Z. Misajleski, Equivalence of intristic shape and shape, God. Zb. Inst. Mat., 42, (2013), 69-80. 
